\title{
Inhibitor of signal transducer and activator of transcription 3 (STAT3) suppresses ovarian cancer growth, migration and invasion and enhances the effect of cisplatin in vitro
}

\author{
Y.J. Tang, Z.L. Sun, W.G. Wu, J. Xing, Y.F. He, D.M. Xin and P. Han \\ Department of Gynecology and Obstetrics, \\ Hebei United University Affiliated Hospital, Tangshan, Hebei Province, China \\ Corresponding author: P. Han \\ E-mail: hanping800@126.com
}

Genet. Mol. Res. 14 (1): 2450-2460 (2015)

Received August 29, 2013

Accepted October 8, 2014

Published March 30, 2015

DOI http://dx.doi.org/10.4238/2015.March.30.3

\begin{abstract}
The aim of the present study was to investigate the antiovarian cancer effect of the inhibitor of signal transducer and activator of transcription 3 (STAT3), WP1066. Western blot was used to detect the phosphorylation of STAT3 in ovarian cancer cell line SKOV3 and cisplatin-resistant ovarian cancer cell line SKOV3/DDP. MTT and colony-forming assays were performed to evaluate the viability and growth of ovarian cancer cells. The apoptosis of ovarian cancer cells was determined by flow cytometry. The wound healing assay and Transwell assay were performed to examine the migration and invasion of ovarian cancer cells. WP1066 significantly inhibited the phosphorylation of STAT3 in SKOV3 and SKOV3/DDP cells. WP1066 treatment inhibited the proliferation and clonogenicity of both SKOV3 and SKOV3/DDP cells. After WP1066 treatment for $24 \mathrm{~h}$, the apoptosis rates of SKOV3 and SKOV3/DDP cells were significantly increased compared with the control cells. After treatment with WP1066, the reduction of the wound gaps was significantly less in both SKOV3 and
\end{abstract}


SKOV3/DDP cells. WP1066 also significantly inhibited the invasion capacity of SKOV3 and SKOV3/DDP cells compared with the control group. Treatment with WP1066 combined with cisplatin significantly increased proliferation inhibition and apoptosis in SKOV3 and SKOV3/ DDP cells compared with treatment with cisplatin alone. A synergistic action between WP1066 and cisplatin on the proliferation and apoptosis of ovarian cancer cells was determined. In conclusion, inhibition of STAT3 may suppress the proliferation, migration and invasion, induce apoptosis and enhance the chemosensitivity of ovarian cancer cells, indicating that STAT3 is a new therapeutic target of ovarian cancer.

Key words: Signal transducer and activator of transcription 3 (STAT3); Inhibitory effect; Ovarian cancer; Cisplatin

\section{INTRODUCTION}

Ovarian cancer is one of the three kinds of malignant tumors of the female reproductive system, with more than 204,000 new cases and 125,000 deaths each year, accounting for $4 \%$ of all cancer cases and $4.2 \%$ of all cancer deaths in women around the world (Xu et al., 2013). Since more than $70 \%$ of patients are diagnosed at an advanced stage, the prognosis of ovarian cancer is very poor. At present, surgery and chemotherapy-based comprehensive therapy are still the main treatment methods for ovarian cancer. Although survival has increased slightly over the past 25 years, 5-year survival remains below 50\% (Corney et al., 2010). In recent years, biotherapies for ovarian cancer, including molecular targeted therapy, immunotherapy and gene therapy, have attracted more and more attention (Zhao et al., 2011; Touboul et al., 2013). Therefore, developing biological methods for ovarian cancer treatment are important for improving the outcome and prognosis of ovarian cancer patients.

Janus protein tyrosine kinase (JAK) is a family of intracellular, nonreceptor tyrosine kinases that transduce cytokine-mediated signals, consisting of four members, JAK1, JAK2, JAK3, and Tyk2 (Ihle, 1995). After binding to cytokines or growth factors, JAK is activated and thereby mediates the signal transducer and activator of transcription (STAT) cascade. The JAK-STAT signaling pathway is widespread in living organisms, and plays important roles not only in the growth of the embryo and stem cells, but also in the occurrence and development of tumors (Quintas-Cardama et al., 2011). STAT3, an intersection of many signaling pathways, not only participates in many physiological and pathological processes, such as inflammation and autophagy, but also plays a vital role in the process of malignant transformation and proliferation of various tumor cells (Buettner et al., 2002; Fossey et al., 2009). In normal cells, the activation of signal transducer and activator of transcription 3 (STAT3) is transient. But in tumor cells, abnormally activated STAT3 upregulates downstream tumor-related genes, such as survivin, Bcl-2, vascular endothelial growth factor (VEGF), and hypoxia-inducible transcription factor 1 (HIF-1), and subsequently promotes tumor cell proliferation, angiogenesis and metastasis, inhibits cell apoptosis, and induces immune escape (Leeman et al., 2006; Johnston and Grandis, 2011). Therefore, blocking the JAK-STAT signaling pathway may inhibit the proliferation, invasion and metastasis and induce the apoptosis of tumor cells, providing a new approach to improve the outcome of ovarian cancer patients. 
WP1066, a small molecule that can selectively block the phosphorylation of STAT3 at tyrosine 705 (Tyr-705) and inhibit the dimer formation of STAT3, is able to inhibit the abnormal activation of STAT3 (Ferrajoli et al., 2007). WP1066 can also decrease immune-inhibitory factor expression such as galectin-3, CCL-2 and sCSFL-1 by inhibiting T cell STAT3 activation, thereby reversing immune suppression (Wei et al., 2011). Although the antitumor effect of WP1066 has been shown in a variety of solid tumors, the effect of WP1066 on ovarian cancer cells has not been investigated. Therefore, we aimed to investigate the antitumor effect of WP1066 on ovarian cancer cells in this study.

\section{MATERIAL AND METHODS}

\section{Cell culture}

Ovarian cancer cell line SKOV3 and cisplatin-resistant ovarian cancer cell line SKOV3/ DDP were cultured in RPMI-1640 medium supplemented with $10 \%$ fetal bovine serum (FBS), $100 \mathrm{U} / \mathrm{mL}$ penicillin and $100 \mu \mathrm{g} / \mathrm{mL}$ streptomycin, at $37^{\circ} \mathrm{C}, 5 \% \mathrm{CO}_{2}$ and $95 \%$ humidity.

\section{MTT assay}

Ovarian cancer cells were seeded on 96-well plates at a density of $1 \times 10^{4}$ cells/well in RPMI-1640 medium supplemented with 10\% FBS. The cells were then treated with or without indicated concentrations of WP1066. Twenty-four hours later, $10 \mu \mathrm{L} 5 \mathrm{mg} / \mathrm{mL}$ MTT solution was added to each well. The plates were incubated at $37^{\circ} \mathrm{C}$ for $4 \mathrm{~h}$, the supernatant was then discarded, and $100 \mu \mathrm{L}$ DMSO was added to each well. The plates were thoroughly mixed before reading on a microplate reader.

\section{Wound healing assay}

Six-well plates were coated with polylysine. SKOV3 and SKOV3/DDP cells were seeded on the coated plates at a density of $1 \times 10^{6} /$ well and grown to $\sim 80 \%$ confluence. The wound healing assay was performed as previously described (Kim et al., 2012). Briefly, a scratch wound was made by scratching with a $10-\mu \mathrm{L}$ pipette tip across the center of the well. After scratching, the well was gently washed twice with medium to remove the detached cells, and RPMI-1640 medium with no serum was added to the wells with or without indicated concentrations of WP1066. Cells were grown at $37^{\circ} \mathrm{C}$ for indicated times and photographs were taken.

\section{Cell invasion assay}

Cell invasion assays were performed using Matrigel matrix-coated Transwell chambers (Costar Corning, Cambridge, MA, USA) according to manufacturer instructions. SKOV3 and SKOV3/DDP cells were serum starved with RPMI-1640 medium for $24 \mathrm{~h}$ and then suspended in serum-free medium. Cells were seeded in the upper wells at a density of $0.5 \times 10^{5} /$ well $(200 \mu \mathrm{L})$. RPMI-1640 medium $(800 \mu \mathrm{L})$ containing 30\% FBS was added to the bottom wells of the plate. Cells were incubated with or without indicated concentrations of WP1066 
for $24 \mathrm{~h}$. Nonmigrating cells were wiped away and the membranes washed with PBS. The membranes were fixed with $4 \%$ paraformaldehyde in PBS and stained with toluidine blue solution. Images were acquired using a Leica DFL290 camera (Leica Microsystems) and analyzed using the Leica application suite software. Migrated cells were counted in nine separate fields per well. The results are representative of three independent experiments.

\section{Colony-forming assay}

Cells were seeded on 6-well plates at $1 \times 10^{3} /$ well per well. Cells were incubated with or without indicated concentrations of WP1066 for 10 days. The cells were then washed twice with PBS, fixed with methanol, and stained with toluidine blue. The colonies were counted under a microscope (Olympus IX81, Tokyo, Japan).

\section{Apoptosis assay}

Annexin V/propidium iodide staining was performed for the detection of apoptotic cells. After a 24-h treatment with or without indicated concentrations of WP1066, the cells were collected and washed twice with ice-cold PBS. The cells were then stained using the annexin $\mathrm{V} /$ propidium iodide for flow cytometry according to manufacturer guidelines.

\section{Western blotting}

Total protein was extracted as previously described (Du et al., 2011). Briefly, the cells were lysed with RIPA lysis buffer (Beyotime, Haimen, Jiangsu, China) and then centrifuged at 12,000-g for $15 \mathrm{~min}$ at $4^{\circ} \mathrm{C}$. The supernatants were collected for subsequent Western blotting. Protein concentration was determined with the BCA method. Proteins were separated by sodium dodecyl sulfate-polyacrylamide gel electrophoresis and transferred to a polyvinylidene fluoride membrane (Millipore, Billerica, MA, USA). The blot was then probed with primary antibody followed by reaction with horseradish peroxidase-conjugated secondary antibody. The signal was detected using enhanced chemiluminescence and recorded on X-ray film.

\section{Statistical analysis}

All the results are reported as means $\pm \mathrm{SD}$. Statistical significance was determined using SPSS 17.0 for Windows. One-way ANOVA was performed for multiple comparisons followed by Fisher LSD post hoc comparisons. Differences were deemed significant if $\mathrm{P}<0.05$.

\section{RESULTS}

\section{Phosphorylation of STAT3 in ovarian cancer cells was suppressed by WP1066}

As shown in Figure 1, after 1.25, 2.5 or $5 \mu \mathrm{M}$ WP1066 treatment for $4 \mathrm{~h}$, the phosphorylation of STAT3 in SKOV3 and SKOV3/DDP cells was significantly suppressed. But $0.625 \mu \mathrm{M}$ WP1066 did not alter the level of p-STAT3. 


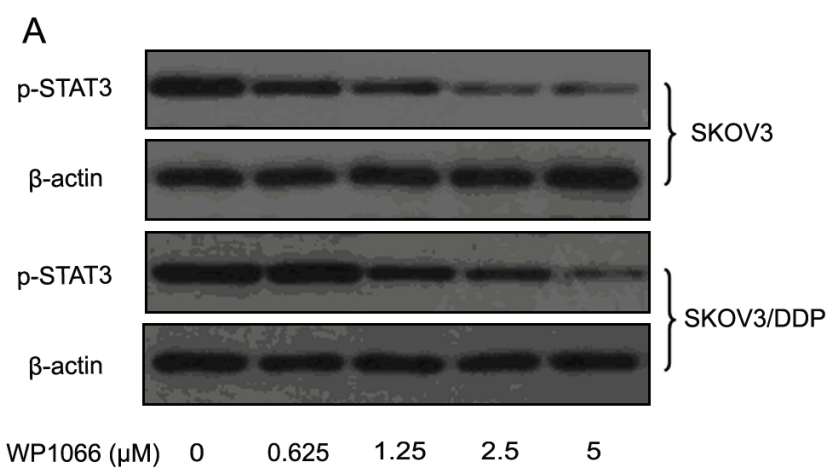

B

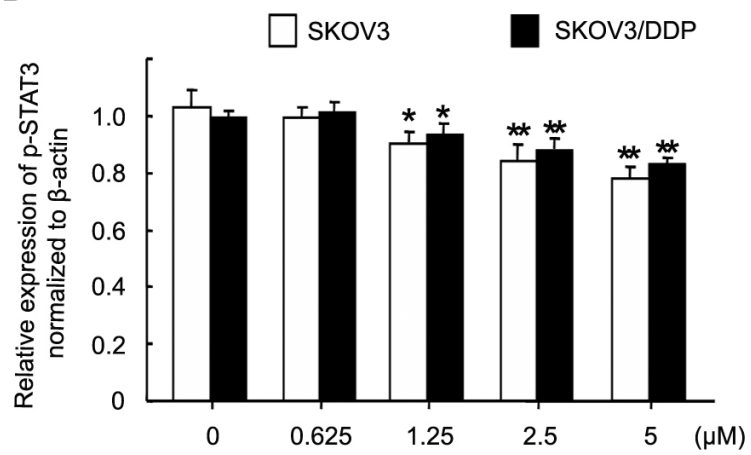

Figure 1. WP1066 inhibited the phosphorylation of STAT3 in ovarian cancer cells. A. Western blot analysis of the p-STAT3 levels in SKOV3 and SKOV3/DDP cells. B. Quantities for densitometric analysis of p-STAT3. Data are reported as means $\pm \mathrm{SD}(\mathrm{N}=3)$. ${ }^{*} \mathrm{P}<0.05, * * \mathrm{P}<0.01$, compared with control.

\section{Proliferation and clonogenicity of ovarian cancer cells were suppressed by WP1066}

Although the phosphorylation of STAT3 in ovarian cancer cells was suppressed by WP1066, whether WP1066 could exert an anti-ovarian cancer effect by suppressing STAT3 phosphorylation was still unclear. Thus, we first investigated the effect of WP1066 on the proliferation of ovarian cancer cells by MTT assay. WP1066 significantly inhibited the proliferation of both SKOV3 and SKOV3/DDP cells at 2.5 and $5 \mu \mathrm{M}$ (Figure 2A). However, there was no significant difference in the inhibitory effect of WP1066 at the same concentration between the two cell lines.

Next, we further determined the effect of WP1066 on the clonogenicity of ovarian cancer cells by the colony-forming assay. As shown in Figure 2B and C, WP1066-treated SKOV3 and SKOV3/DDP cells exhibited much fewer and smaller colonies compared with control.

\section{WP1066 induced the apoptosis of ovarian cancer cells}

Next, we further investigated whether WP1066 treatment could induce the apoptosis of ovarian cancer cells. After WP1066 treatment for $24 \mathrm{~h}$, the apoptosis rates of SKOV3 and SKOV3/DDP cells were both significantly increased compared with control (Figure 3). How- 
ever, there was no significant difference in the apoptosis rate between SKOV3 and SKOV3/ DDP cells.
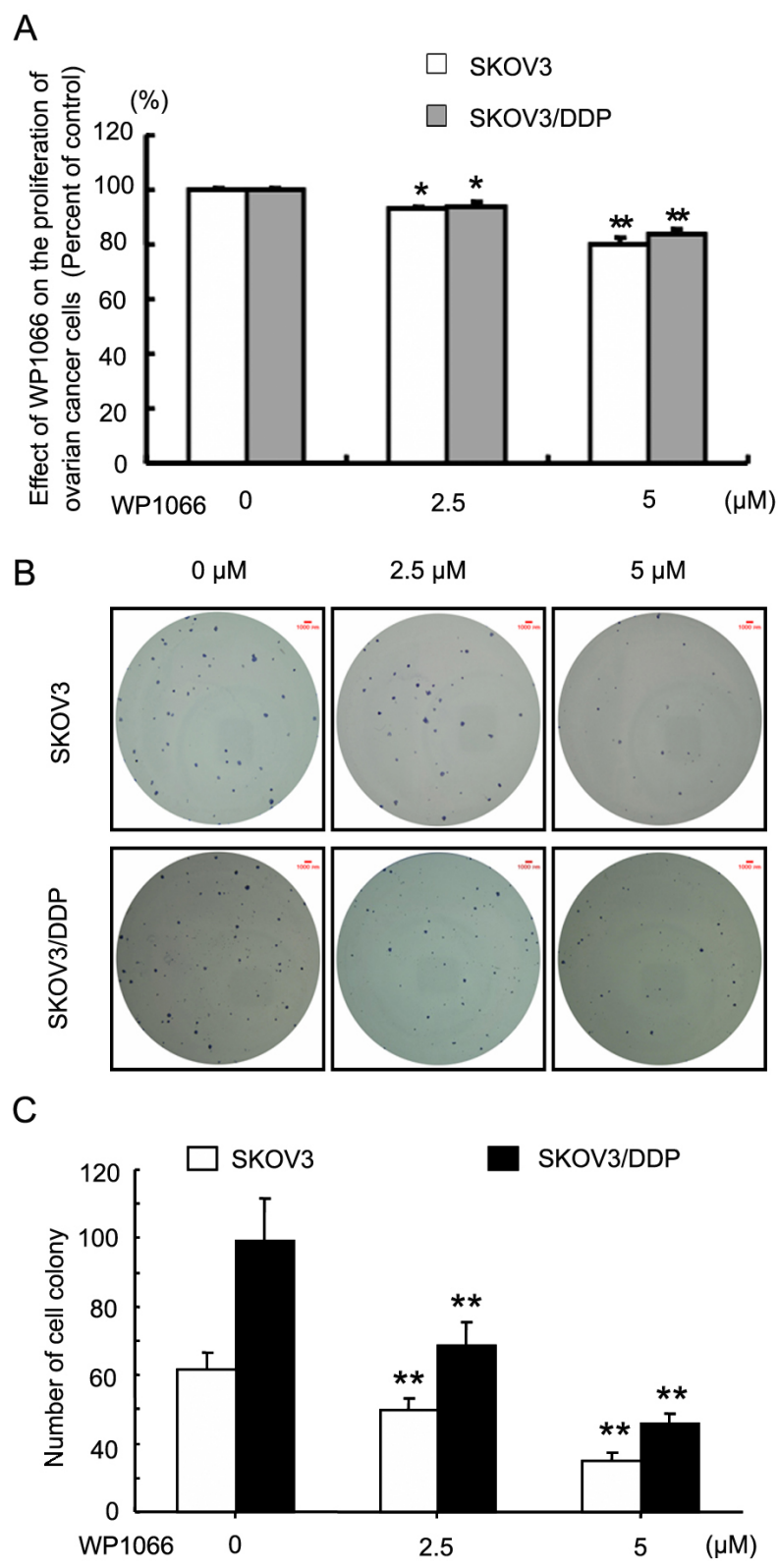

Figure 2. WP1066 inhibited the proliferation and colony formation of ovarian cancer cells. A. Effect of WP1066 on the proliferation of ovarian cancer cells. B. Effect of WP1066 on the colony formation of ovarian cancer cells. C. Number of cell colony. Cells were seeded on 6-well plates and incubated with or without WP1066 for 10 days. Then the number of colony was counted under the microscope. The results are representative of three independent experiments. Data are reported as means $\pm \mathrm{SD} . * \mathrm{P}<0.05$, $* * \mathrm{P}<0.01$, compared with control. 


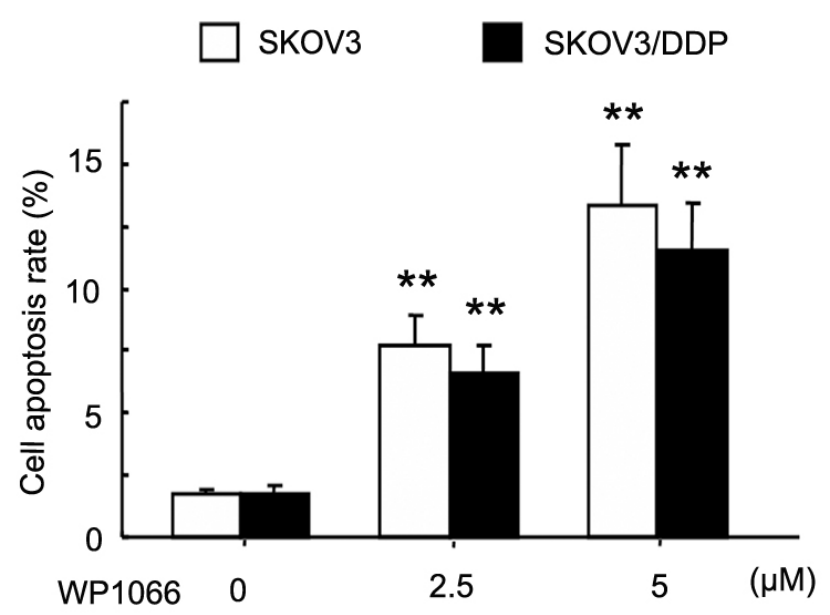

Figure 3. Effect of WP1066 on the apoptosis of ovarian cancer cells. Ovarian cancer cells were treated with WP1066 for $24 \mathrm{~h}$ and then the apoptosis was analyzed by flow cytometry. Data are reported as means \pm SD. $* \mathrm{P}<$ $0.05, * * \mathrm{P}<0.01$, compared with control.

\section{Migration and invasion of ovarian cancer cells were suppressed by WP1066}

Since the above experiments had shown that the inhibition of STAT3 could inhibit the proliferation of ovarian cancer cells and induce their apoptosis, we wondered whether WP1066 could also inhibit the migration of ovarian cancer cells. In the control group, the wound gaps were significantly less compared with the initial gaps (Figure 4A and B). The wound gap of SKOV3/DDP cells was clearly less than that of SKOV3 cells, indicating that SKOV3/DDP cells had a higher migration rate than did the SKOV3 cells. After treatment with WP1066, the reduction of the wound gaps was significantly less in both SKOV3 and SKOV3/ DDP cells, indicating that WP1066 may inhibit the migration of ovarian cancer cells.

To further study whether WP1066 could inhibit the invasion of ovarian cancer cells, we performed Transwell tumor cell invasion assays. After WP1066 treatment, the numbers of SKOV3 and SKOV3/DDP cells passing through the membrane were significantly decreased, suggesting that WP1066 could inhibit the invasion capacity of both normal and cisplatinresistant ovarian cancer cells (Figure 4C and D).

\section{WP1066 enhanced the effect of cisplatin on ovarian cancer cells}

To investigate whether STAT3 inhibition could enhance the anti-ovarian cancer effect of cisplatin, we observed the viability and apoptosis of ovarian cancer cells after combined WP1066 and cisplatin treatment. As shown in Figure 5A, treatment of SKOV3 cells with WP1066 in combination with cisplatin showed greater proliferation inhibition compared to treatment with cisplatin alone at each indicated concentration. Similar results were obtained in SKOV3/DDP cells (Figure 5B). The q values calculated with the Jin Zhengjun method were $1.40,1.38$ and 1.34 for the combination of $2.5 \mu \mathrm{M}$ WP1066 and $1.25,2.5$ and $5 \mu \mathrm{g} / \mathrm{mL}$ cisplatin, respectively. Combined WP1066 and cisplatin also significantly increased the apoptosis 


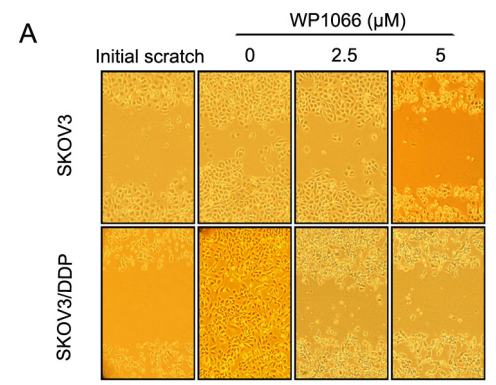

B

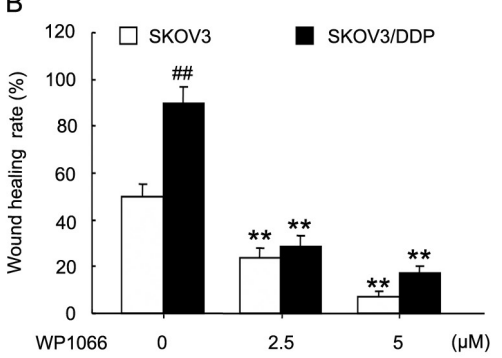

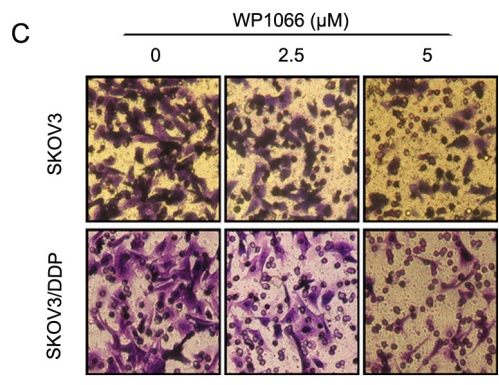

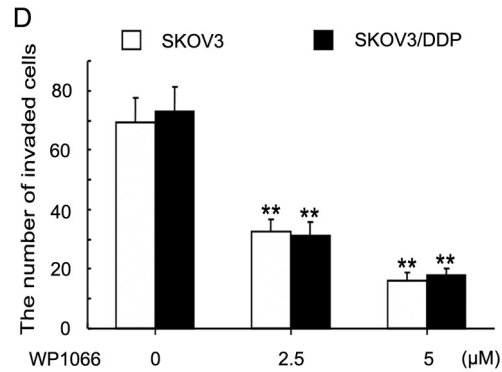

Figure 4. WP1066 inhibited the migration (A. and B.) and invasion (C. and D.) of ovarian cancer cells. Cells were seeded on 6-well plates. After $24 \mathrm{~h}$, scratch wounds were generated as described in Method. Then the cells were treated with WP1066 for another $24 \mathrm{~h}$. Images were taken and the wound gaps were determined. The initial length of wound gap was calculated as $100 \%$. Percent of wound contraction was calculated taking the initial size of the wound gap as $100 \%$. Cell invasion assays were performed using Matrigel matrix coated Transwell chambers as described in Method. Migrated cells were counted in nine separated fields per well. The results are representative of three independent experiments. ${ }^{*} \mathrm{P}<0.05,{ }^{*} \mathrm{P}<0.01$, compared with untreated cells. ${ }^{\#} \mathrm{P}<0.01$, compared with untreated SKOV3 cells.
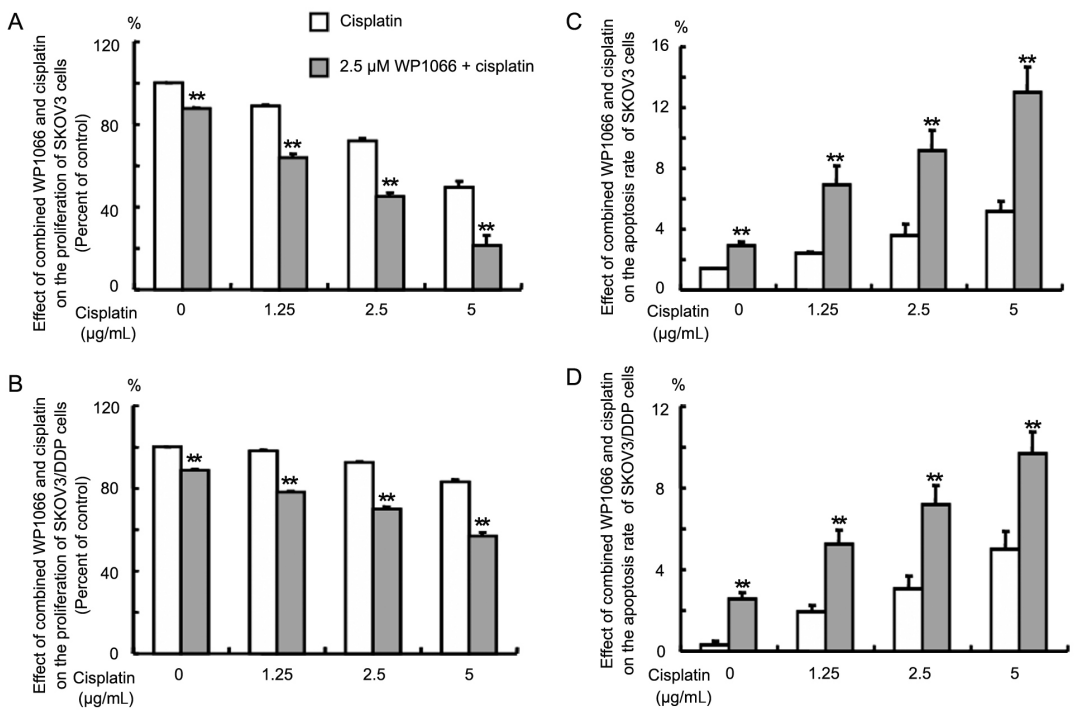

Figure 5. WP1066 enhanced the effect of cisplatin on the proliferation (A. and B.) and apoptosis (C. and D.) of ovarian cancer cells. SKOV3 and SKOV3/DDP cells were treated with cisplatin alone or combined with $2.5 \mu \mathrm{M}$ WP1066 for $24 \mathrm{~h}$. Then, the proliferation and apoptosis were determined by MTT and flow cytometry. Data are reported as means $\pm \mathrm{SD} . * \mathrm{P}<0.05, * * \mathrm{P}<0.01$, compared with control. 
rate of both SKOV3 and SKOV3/DDP cells (Figure 5C and D). The q values for WP1066/1.25 $\mu \mathrm{g} / \mathrm{mL}$ cisplatin, WP1066/2.5 $\mu \mathrm{g} / \mathrm{mL}$ cisplatin and WP1066 $/ 5 \mu \mathrm{g} / \mathrm{mL}$ cisplatin were $1.17,1.28$ and 1.30, respectively. These results indicated that there was a synergistic action between WP1066 and cisplatin on the proliferation and apoptosis of ovarian cancer cells.

\section{DISCUSSION}

It has been reported that the overexpression of STAT3 in ovarian tumors is associated with ovarian tumorigenesis (Rosen et al., 2006). In the present study, the results showed that WP1066 was able to inhibit the phosphorylation of STAT3 and subsequently inhibit the growth, colony formation, migration, and invasion of ovarian cancer cells. WP1066 also induced the apoptosis of ovarian cancer cells. In addition, WP1066 increased the chemosensitivity of both SKOV3 and SKOV3/DDP cells to cisplatin.

Inactivated STAT3 is located in the cytoplasm. When Tyr-705 is phosphorylated, the activated STAT3 dimerizes and translocates into the nucleus. It has been widely documented that sustained activation of STAT3 can induce abnormal upregulation of tumor-related genes (Zhang et al., 2012) and participate in tumor cell proliferation, apoptosis, immune escape, angiogenesis, invasion, and metastasis (Ozols et al., 2006; Liu et al., 2013). Currently, STAT3 has been studied as a tumor therapeutic target (Leeman et al., 2006). Several small molecule inhibitors of STAT3 have shown certain potential in the targeted therapy of tumor (Chen et al., 2013; Kaneko et al., 2013). WP1066 is able to block the phosphorylation of JAK2 kinases and STAT3 protein and has shown antitumor properties in renal carcinoma, lymphoma and many other tumors (Horiguchi et al., 2010; Hatiboglu et al., 2012). Therefore, WP1066 was used to test its anti-ovarian cancer capacity in the present study.

The proliferation and apoptosis-related genes, such as Bcl-2, cyclin B1 and cyclin D1, are downstream genes of the JAK-STAT3 pathway (Iwamaru et al., 2007; Wei et al., 2011). In ovarian cancer cells with excessive activation of STAT3 and overexpression of multidrugresistance genes, reduction of STAT3 phosphorylation down-regulates the expression of inhibitors of apoptosis, such as survivin, Bcl-2 and Bcl-xl, and then inhibits the proliferation and promotes the apoptosis of ovarian cancer cells (Duan et al., 2009). In this study, we showed that WP1066 decreased the p-STAT3 levels in both SKOV3 and SKOV3/DDP cells. WP1066 inhibited the proliferation and induced the apoptosis of ovarian cancer cells. Furthermore, in the present study, we also examined the colony formation of ovarian cancer cells, which may reflect the population dependence and proliferation ability of the cells. The results showed that SKOV3/DDP cells have a higher colony-forming ability than do SKOV3 cells, indicating that SKOV3/DDP cells have better viability and lower population dependence. Inhibition of p-STAT3 with WP1066 also significantly decreased the colony-forming ability of ovarian cancer cells.

Invasion of surrounding tissues and distant metastasis are two important hallmarks of malignant tumors, which seriously affect treatment success and prognosis of the patients. Thus, we further tested the migration and invasion ability of ovarian cancer cells by the wound healing assay and Transwell assay. Our results showed that SKOV3/DDP cells had stronger migration and invasion ability compared with SKOV3 cells. Inhibition of p-STAT3 by WP1066 suppressed the migration and invasion of the ovarian cancer cells. STAT3 is able to activate the expression of cyclin D1 and survivin in smooth muscle cells, promotes the formation of neointimal cells and thus the proliferation and migration of smooth muscle cells (Daniel et al., 
2012). Overexpression of STAT3 and IL-6 could induce the expression of HIF-1 $\alpha$ and VEGF (Yang et al., 2013). Blockade of p-STAT3 can reduce the expression of HIF- $\alpha$, VEGF, MMP2, and MMP-9, and inhibit the proliferation and invasion of carcinoma cells (Messina et al., 2008; Vermeij et al., 2008; Boreddy et al., 2011; Yan et al., 2012). Our results demonstrated that STAT3 inhibition decreases the metastatic potential of ovarian cancer cells.

Cisplatin, used as a frontline agent in the treatment of advanced ovarian cancer (Hess et al., 2007), is usually associated with chemoresistance (Parker et al., 1991). It has been recognized that the overexpression and activation of STAT3 are associated with chemoresistance to cisplatin (Duan et al., 2006; Dijkgraaf et al., 2013). Our results showed that WP1066 enhanced the anti-tumor capacity of cisplatin in SKOV3 ovarian cancer cells. More interestingly, WP1066 also improved the effect of cisplatin on SKOV3/DDP cell proliferation and apoptosis. A synergistic action was noted between WP1066 and cisplatin. These results suggest that WP1066 is able to increase the chemosensitivity of ovarian cancer cells to cisplatin and reverse the cisplatin resistance of ovarian cancer cells.

In summary, WP1066 can inhibit the activation of STAT3 and thereby regulate the downstream expression of tumor-related genes. Our results demonstrated that WP1066 could suppress proliferation, migration and invasion and induce apoptosis in ovarian cancer cells. WP1066 could also enhance chemosensitivity in both general and cisplatin-resistant ovarian cancer cells. Our data indicate that STAT3 is a new therapeutic target of ovarian cancer. However, this is just an in vitro study. Further in vivo experiments should be performed in the future to verify the anti-ovarian cancer effect of WP1066.

\section{Conflicts of interest}

The authors declare no conflict of interest.

\section{REFERENCES}

Boreddy SR, Sahu RP and Srivastava SK (2011). Benzyl isothiocyanate suppresses pancreatic tumor angiogenesis and invasion by inhibiting HIF-alpha/VEGF/Rho-GTPases: pivotal role of STAT-3. PLoS One 6: e25799.

Buettner R, Mora LB and Jove R (2002). Activated STAT signaling in human tumors provides novel molecular targets for therapeutic intervention. Clin. Cancer Res. 8: 945-954.

Chen X, Du Y, Nan J, Zhang X, et al. (2013). Brevilin A, a novel natural product, inhibits janus kinase activity and blocks STAT3 signaling in cancer cells. PLoS One 8: e63697.

Corney DC, Hwang CI, Matoso A, Vogt M, et al. (2010). Frequent downregulation of miR-34 family in human ovarian cancers. Clin. Cancer Res. 16: 1119-1128.

Daniel JM, Dutzmann J, Bielenberg W, Widmer-Teske R, et al. (2012). Inhibition of STAT3 signaling prevents vascular smooth muscle cell proliferation and neointima formation. Basic Res. Cardiol. 107: 261.

Dijkgraaf EM, Heusinkveld M, Tummers B, Vogelpoel LT, et al. (2013). Chemotherapy alters monocyte differentiation to favor generation of cancer-supporting M2 macrophages in the tumor microenvironment. Cancer Res. 73: 2480-2492.

Du J, Cheng B, Zhu X and Ling C (2011). Ginsenoside Rg1, a novel glucocorticoid receptor agonist of plant origin, maintains glucocorticoid efficacy with reduced side effects. J. Immunol. 187: 942-950.

Duan Z, Foster R, Bell DA, Mahoney J, et al. (2006). Signal transducers and activators of transcription 3 pathway activation in drug-resistant ovarian cancer. Clin. Cancer Res. 12: 5055-5063.

Duan Z, Ames RY, Ryan M, Hornicek FJ, et al. (2009). CDDO-Me, a synthetic triterpenoid, inhibits expression of IL-6 and Stat3 phosphorylation in multi-drug resistant ovarian cancer cells. Cancer Chemother. Pharmacol. 63: 681-689.

Ferrajoli A, Faderl S, Van Q, Koch P, et al. (2007). WP1066 disrupts Janus kinase-2 and induces caspase-dependent apoptosis in acute myelogenous leukemia cells. Cancer Res. 67: 11291-11299.

Fossey SL, Liao AT, McCleese JK, Bear MD, et al. (2009). Characterization of STAT3 activation and expression in canine 
and human osteosarcoma. BMC Cancer 9: 81.

Hatiboglu MA, Kong LY, Wei J, Wang Y, et al. (2012). The tumor microenvironment expression of p-STAT3 influences the efficacy of cyclophosphamide with WP1066 in murine melanoma models. Int. J. Cancer 131: 8-17.

Hess LM, Benham-Hutchins M, Herzog TJ, Hsu CH, et al. (2007). A meta-analysis of the efficacy of intraperitoneal cisplatin for the front-line treatment of ovarian cancer. Int. J. Gynecol. Cancer 17: 561-570.

Horiguchi A, Asano T, Kuroda K, Sato A, et al. (2010). STAT3 inhibitor WP1066 as a novel therapeutic agent for renal cell carcinoma. Br. J. Cancer 102: 1592-1599.

Ihle JN (1995). The Janus protein tyrosine kinase family and its role in cytokine signaling. Adv. Immunol. 60: 1-35.

Iwamaru A, Szymanski S, Iwado E, Aoki H, et al. (2007). A novel inhibitor of the STAT3 pathway induces apoptosis in malignant glioma cells both in vitro and in vivo. Oncogene 26: 2435-2444.

Johnston PA and Grandis JR (2011). STAT3 signaling: anticancer strategies and challenges. Mol. Interv. 11: 18-26.

Kaneko N, Kita A, Yamanaka K and Mori M (2013). Combination of YM155, a survivin suppressant with a STAT3 inhibitor: a new strategy to treat diffuse large B-cell lymphoma. Leuk. Res. 37: 1156-1161.

Kim M, Yoon S, Lee S, Ha SA, et al. (2012). Gremlin-1 induces BMP-independent tumor cell proliferation, migration, and invasion. PLoS One 7: e35100.

Leeman RJ, Lui VW and Grandis JR (2006). STAT3 as a therapeutic target in head and neck cancer. Expert Opin. Biol. Ther. 6: 231-241.

Liu K, Jiang M, Lu Y, Chen H, et al. (2013). Sox2 cooperates with inflammation-mediated Stat 3 activation in the malignant transformation of foregut basal progenitor cells. Cell Stem Cell 12: 304-315.

Messina JL, Yu H, Riker AI, Munster PN, et al. (2008). Activated stat-3 in melanoma. Cancer Control 15: 196-201.

Ozols RF, Bookman MA, du Bois A, Pfisterer J, et al. (2006). Intraperitoneal cisplatin therapy in ovarian cancer: comparison with standard intravenous carboplatin and paclitaxel. Gynecol. Oncol. 103: 1-6.

Parker RJ, Eastman A, Bostick-Bruton F and Reed E (1991). Acquired cisplatin resistance in human ovarian cancer cells is associated with enhanced repair of cisplatin-DNA lesions and reduced drug accumulation. J. Clin. Invest. 87: $772-777$.

Quintas-Cardama A, Kantarjian H, Cortes J and Verstovsek S (2011). Janus kinase inhibitors for the treatment of myeloproliferative neoplasias and beyond. Nat. Rev. Drug Discov. 10: 127-140.

Rosen DG, Mercado-Uribe I, Yang G, Bast RC Jr, et al. (2006). The role of constitutively active signal transducer and activator of transcription 3 in ovarian tumorigenesis and prognosis. Cancer 107: 2730-2740.

Touboul C, Lis R, Al Farsi H, Raynaud CM, et al. (2013). Mesenchymal stem cells enhance ovarian cancer cell infiltration through IL6 secretion in an amniochorionic membrane based 3D model. J. Transl. Med. 11: 28.

Vermeij J, Teugels E, Bourgain C, Xiangming J, et al. (2008). Genomic activation of the EGFR and HER2-neu genes in a significant proportion of invasive epithelial ovarian cancers. BMC Cancer 8: 3.

Wei J, Wu A, Kong LY, Wang Y, et al. (2011). Hypoxia potentiates glioma-mediated immunosuppression. PLoS One 6: e16195.

Xu YZ, Xi QH, Ge WL and Zhang XQ (2013). Identification of serum microRNA-21 as a biomarker for early detection and prognosis in human epithelial ovarian cancer. Asian Pac. J. Cancer Prev. 14: 1057-1060.

Yan N, Zhang S, Yang Y, Cheng L, et al. (2012). Therapeutic upregulation of Class A scavenger receptor member 5 inhibits tumor growth and metastasis. Cancer Sci. 103: 1631-1639.

Yang F, Zhang W, Li D and Zhan Q (2013). Gadd45a suppresses tumor angiogenesis via inhibition of the mTOR/STAT3 protein pathway. J. Biol. Chem. 288: 6552-6560.

Zhang Q, Gong R, Qu J, Zhou Y, et al. (2012). Activation of the Ras/Raf/MEK pathway facilitates hepatitis C virus replication via attenuation of the interferon-JAK-STAT pathway. J. Virol. 86: 1544-1554.

Zhao SH, Zhao F, Zheng JY, Gao LF, et al. (2011). Knockdown of stat3 expression by RNAi inhibits in vitro growth of human ovarian cancer. Radiol. Oncol. 45: 196-203. 